\title{
Future Developments in Clinical MEG and Its Combination with nTMS
}

\section{Mäkelä, Jyrki}

Springer-Verlag

2014

Mäkelä , J 2014 , Future Developments in Clinical MEG and Its Combination with nTMS . in S Supek \& C J Aine (eds), Magnetoencephalography : From Signals to Dynamic Cortical Networks . V edn , Springer-Verlag , pp. 933-938 . https://doi.org/10.1007/978-3-642-33045-2_45

http://hdl.handle.net/10138/152850

https://doi.org/10.1007/978-3-642-33045-2_45

publishedVersion

Downloaded from Helda, University of Helsinki institutional repository.

This is an electronic reprint of the original article.

This reprint may differ from the original in pagination and typographic detail.

Please cite the original version. 


\title{
Future Developments in Clinical MEG and Its Combination with nTMS
}

\author{
Jyrki P. Mäkelä
}

\begin{abstract}
Development of clinical MEG will provide biomarkers of neurodegenerative disorders by producing functional and effective connectivity measures within and between distinct functional brain areas. It is highly probable that neurodegenerative disorders damage these connections early in their course and detection of such changes will be feasible with sophisticated signal analysis of MEG data. Combining MEG and nTMS has already proven to be valuable in clinical evaluations. Such combinations will assist us in understanding the complex brain networks and the effective connectivity within them both in the healthy and diseased brains.
\end{abstract}

Keywords Connectivity - Epilepsy - Neurodegenerative diseases - Navigated transcranial magnetic stimulation (nTMS)

\section{MEG in Clinical Connectivity Studies}

The trend in the MEG community, as well as in the neuroscience community in general, is to reveal the brain functions creating the unified perceptions of the world, despite the parcellated presentation of its features in our brains. Higher level cognitive functions such as attention, working memory and sensory awareness also arise from activations in widespread cortical networks. The complete view/model of these functional networks will require understanding of anatomical, functional and effective connectivity within and between distinct functional brain areas. MEG, with its excellent temporal and tolerable spatial accuracy will definitively play an important role in this endeavor (e.g., Palva et al. 2010; Hipp et al. 2012).

\footnotetext{
J. P. Mäkelä (ه)

BioMag Laboratory, HUS Medical Imaging Center, Helsinki University Central Hospital, P.O. Box 340, 00029 Helsinki, Finland

e-mail: jyrki.makela@hus.fi
} 
The advances of neuroscience and clinical applications of MEG have been linked closely to progress of instrumentation and signal analysis methods. Development of instrumentation provides new possibilities as "hypothesis generating" research, complementing the traditional "hypothesis testing" approaches.

Studies of signal conduction between different brain areas using MEG were first started in patients with epilepsy. Already the early efforts demonstrated that MEG is able to identify source locations of epileptiform activity and map its spread to the opposite hemisphere (Barth et al. 1982). More recent studies have convincingly shown the usefulness of MEG studies in planning epilepsy surgery. MEG improves the treatment plan in about $20-30 \%$ of the patients (Sutherling et al. 2008; Knowlton et al. 2009; de Tiege et al. 2012). However, source localization of the earliest epileptiform activity, not a detailed analysis of its spread, has been the main target of MEG studies in epilepsy (for a review, see, e.g., Mäkelä et al. 2006). The significance of tracking the spread of epileptiform activity may increase along with developments of epilepsy surgery planning, e.g., in increased use of stereotactic EEG as opposed to subdural grid recordings.

Recent developments have made studies of such "clinical connectivity" more precise. New, more comfortable gantries and continuous head position localization have made ictal MEG recordings more convenient, and they localize ictal onset zone with high sensitivity and specificity at the brain lobe level. Sources of ictal onset MEG signals and interictal dipole clusters are essentially equally specific in estimation of the ictal onset zone as defined by ECoG, but ictal MEG is more sensitive (Medvedovsky et al. 2012). A combination of MEG with precisely time-locked video recordings has enhanced the identification of ictal events and eased the recognition of artifacts (Zhdanov et al. 2013). These applications, developed in clinical studies, will also definitively assist sophisticated basic research experiments, e.g., by guiding the data analysis into precisely selected time windows of required behaviors and provide additional information for MEG "metadata" storage.

MEG may also provide tools to improve diagnostics of neurodegenerative disorders. In these conditions, detection of functional disconnection between brain regions will be crucial. For example, early $\mathrm{AD}$ pathology results in abnormal interactions between neuronal systems even before the onset of clinical signs and symptoms (Delbeuck et al. 2003; Brier et al. 2012). MEG is a useful tool to identify a "signature" of altered functional connectivity that can distinguish pathological processes from normal cognition (Stam et al. 2009). MEG studies may provide unique information regarding the changes in brain function responsible for the development of clinical dementia. This should help to direct the development of treatment strategies (e.g., as an endpoint in clinical trials), and in the tracking of disease progression. Because MEG is sensitive to dendritic activity at the synaptic level (Murakami and Okada 2006), it may be able to detect pathology even before there is evidence of other "positive" neuroimaging biomarkers (e.g., in vivo amyloid imaging; for a review, see Zamrini et al. 2011).

The present connectivity analysis methods require relatively long recordings of high-quality signals for providing meaningful results. Exquisite experimental setups are needed to avoid problems related to varying vigilance. Moreover, 
sophisticated movement correction and artifact suppression are required for complete realization of their clinical value. Fortunately, MEG noise suppression methods have developed rapidly. The signal space separation algorithm (SSS) allows the recognition of magnetic signals from different subspaces, e.g., from the head and its surroundings (Taulu and Simola 2006). The removal of the signals that appear statistically similar in both subspaces strongly suppresses the artifacts generated even in the close vicinity of the sensors, e.g., by electric stimulation of subthalamic electrodes in patients with Parkinson's disease. This expands the MEG applications into studies of effects of deep brain stimulation on spontaneous brain activity in different neurodegenerative diseases (Airaksinen et al. 2012). The present efficacy of the SSS method can probably be enhanced further by optimizing the MEG sensor array to also include elements measuring the tangential components of the extracranial magnetic field (Nurminen et al. 2013). Besides external noise, random sensor noise may also deteriorate data quality. It is possible to use SSS for simultaneous modeling of the correlated signals from the brain and magnetic interference, and the uncorrelated part (from sensor noise) of a multichannel MEG signal, and thus aid in removing the uncorrelated part from the source estimation. This approach decreases the white noise level with a factor of about 2-4 while the physiological spectral peaks remain intact (Taulu et al. 2012). This is particularly important in single-trial analysis of evoked responses, and in analyzing high-frequency signals having relatively poor signal-to noise ratio. The method may also have clinical relevance e.g., in detecting high-frequency epileptiform signals (Helle et al. 2012). These developments will definitely assist in obtaining more crisp data for connectivity analyses, and also aid in applying the new analysis methods in the clinical diagnostics.

Time will tell, whether new MEG analysis methods searching for corticocortical spatial (Schnitzler and Gross 2005), phase-related (Palva et al. 2010), and temporal correlations (Montez et al. 2009) of spontaneous MEG networks in signal or source spaces will produce robust biomarkers of disease in individual patients. The complex methods used in data mining and complicated statistics associated with them may be relatively impenetrable for clinical users. In order to further the integration of clinical MEG results into routine patient flow, the analyses also need to be fast and understandable to the clinical teams utilizing them. The hypotheses and presumptions underlying the modeling need to be clear, and the effects of various details of the models required for the completion of the final results need to be thoroughly understood. Clinical decision making is seldom based on one methodology only. Nevertheless, developers of new analysis methods for such purposes may benefit by placing themselves into the clinical situation, i.e., as a target for the planned procedures, or to consider their willingness to use preventive medication for the next 20 years, based on data analysis results conducted by a technician. Although solving such usability problems is not necessarily attractive for researchers in basic neuroscience, it is highly important in clinical research and particularly in MEG clinical applications. 


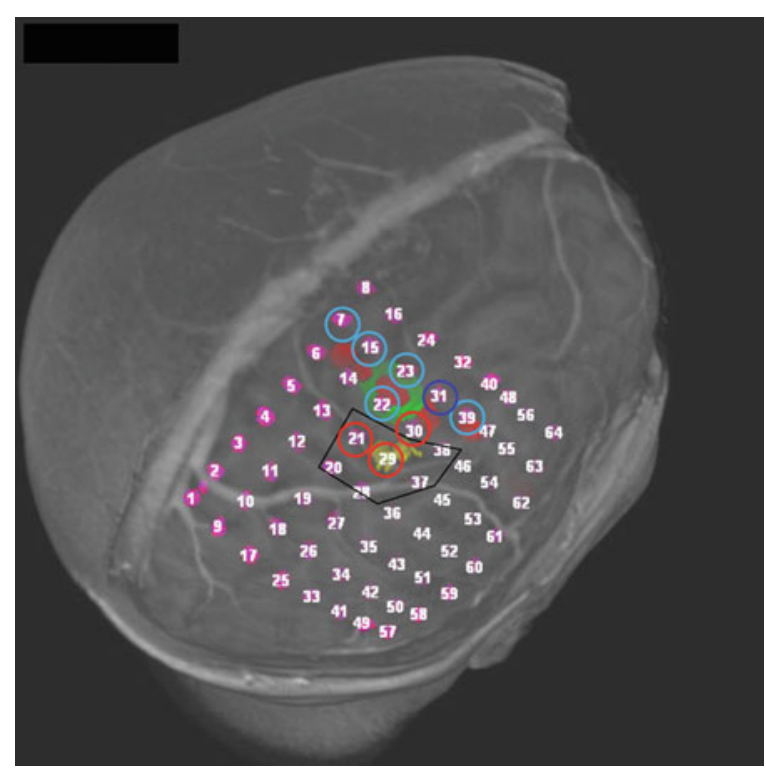

Fig. 1 Comparison of mapping with MEG, nTMS and ECoG in a patient with epilepsy, depicted on a 3-D reconstruction of the patient's brain. Epileptiform region near the motor cortex, as depicted by MEG, is colored yellow. Red dots indicate sites producing motor evoked potentials in nTMS. Green dot indicates the anatomic indicator of the hand motor area. Red circles mark electrodes where stimulation elicited typical seizures, dark blue circle indicates site producing hand movements and a seizure, and light blue circles indicate sites producing hand and arm movements. The surgeon removed the cortical area delineated by black lines. After the operation, the patient has remained free of seizures. Modified from Vitikainen et al. (2009)

\section{Combination of MEG with Navigated Transcranial Magnetic Stimulation}

The physics underlying noninvasive transcranial magnetic stimulation (TMS) can be considered as the reverse of MEG; instead of picking up tiny magnetic signals from the cortex, it utilizes fast, strong (about $2 \mathrm{~T}$ ) magnetic pulses to modify cortical activity. Navigated TMS (nTMS) displays a dynamic estimate of the stimulus-induced electric field on the patient's individual 3-D brain MRI reconstruction, and enables selection of localized stimulation targets from it. The effects of nTMS can be tested on the source areas selected from MEG. For example, nTMS delivered to secondary somatosensory cortex area, pinpointed by MEG, speeds up somatomotor reactivity (Raij et al. 2008), and rhythmic TMS to the MEG-identified source areas of spontaneous oscillatory activity entrains these oscillations at the stimulation frequency (Thut et al. 2011) (Fig. 1).

In the foreseeable future, TMS devices will develop towards more complex delivery of pulses into multiple sites, monitoring the effects of TMS by electrophysiological measures, and even guiding the TMS properties by the induced 
modifications. Although simultaneous TMS and MEG recordings probably will not be feasible, MEG will be a crucial tool in interpreting the electrophysiological connectivity utilized in such studies. Combining MEG and nTMS has already proven to be valuable in clinical evaluations (e.g. Vitikainen et al. 2009; Mäkelä et al. 2013, see also Fig. 1). Such combinations will assist us in understanding the complex brain networks, the effective connectivity within them both in the healthy and diseased brains.

\section{References}

Airaksinen K, Butorina A, Pekkonen E, Nurminen J, Taulu S, Ahonen A, Schnitzler A, Mäkelä JP (2012) Somatomotor mu rhythm amplitude correlates with rigidity during deep brain stimulation in Parkinsonian patients. Clin Neurophysiol 123:2010-2017

Barth DS, Sutherling W, Engel J Jr, Beatty J (1982) Neuromagnetic localization of epileptiform spike activity in the human brain. Science 218:891-894

Brier MR, Thomas JB, Snyder AZ, Benzinger TL, Zhang D, Raichle ME, Holltzman DM, Morris JC, Ances BM (2012) Loss of intranetwork and internetwork resting state functional connections with Alzheimer disease progression. J Neurosci 32:8890-8899

Delbeuck X, van der Linden M, Collette F (2003) Alzheimer's disease as disconnection syndrome? Neuropsychol Rev 13:79-91

DeTiege X, Carrette E, Legros B, Vonck K, Op de Beeck M, Bourguignon M, Massager N, David P, Van Roost D, Meurs A, Lapere S, Deblaere K, Goldman S, Van Bogaert P (2012) Clinical added value of magnetic source imaging in the presurgical evaluation of refractory focal epilepsy. J Neurol Neurosurg Psychiatry 83:417-423

Helle L, Parkkonen L, Taulu S, Ahonen A (2012) Suppression of uncorrelated sensor noise and artifacts: demonstration with high-frequency brain signals. Poster in Biomag 2012, Paris

Hipp JF, Hawellek DJ, Corbetta M, Siegel M, Engel AK (2012) Large-scale cortical correlation structure of spontaneous oscillatory activity. Nat Neurosci 15:884-890

Knowlton RC, Radzan SN, Limdi N, Elgavish RA, Killen J, Blount J, Burneo JG, Ver Hoef L, Paige L, Faught E, Kankiratwana P, Bartolocci A, Riley K, Kusniwecky R (2009) Effect of epilepsy magnetic source imaging on intracranial electrode placement. Ann Neurol 65:716-723

Medvedovsky M, Taulu S, Gaily E, Metsähonkala E-L, Mäkelä JP, Ekstein D, Kipervasser S, Neufeld MY, Kramer U, Blomstedt G, Fried I, Karppinen A, Veshchev I, Roivainen R, Ben-Zeev B, Goldberg-Stern H, Wilenius J, Paetau R (2012) Sensitivity and specificity of seizure onset zone estimation by ictal MEG. Epilepsia 53:1649-1657

Montez T, Poil S-S, Jones BF, Manshanden I, Verbunt JPA, van Dijk P, Brussaard AB, van Ooyen A, Stam CJ, Scheltens P, Linkenkaer-Hansen K (2009) Altered temporal correlations in parietal alpha and prefrontal theta oscillations in early-stage Alzheimer disease. PNAS 106:1614-1619

Murakami S, Okada Y (2006) Contributions of principal neocortical neurons to magnetoencephalography and electroencephalography signals. J Physiol 575(3):925-936

Mäkelä JP, Forss N, Jääskeläinen J, Kirveskari E, Korvenoja A, Paetau R (2006) Magnetoencephalography in neurosurgery. Neurosurgery 59:493-510

Mäkelä JP, Vitikainen A-M, Lioumis P, Paetau R, Ahtola E, Kuusela L, Valanne L, Blomstedt G, Gaily E (2013) Functional plasticity of the motor cortical structures demonstrated by navigated TMS in two patients with epilepsy. Brain Stimulat 6:286-291

Nurminen J, Taulu S, Nenonen J, Helle L, Simola J, Ahonen A (2013) Improving MEG performance with additional tangential sensors. IEEE Trans Biomed Eng 60:2559-2566 
Palva JM, Monto S, Kulashekrar S, Palva S (2010) Neuronal synchrony reveals working memory networks and predicts individual memory capacity. PNAS 107:7580-7585

Raij T, Karhu J, Kičić D, Lioumis JP, Lin F-H, Ahveninen J, Ilmoniemi RJ, Mäkelä JP, Hämäläinen M, Rosen BR, Belliveau JW (2008) Parallel sensory input makes the brain run faster. NeuroImage 40:1792-1797

Schnitzler A, Gross J (2005) Normal and pathological oscillatory communication in the brain. Nat Neurosci 6:285-296

Stam CJ, de Haan W, Daffertshofer A, Jones BF, Manshanden I, van Cappellen van Walsum AM, Montez T, Verbunt JP, de Munck JC, van Dijk BW, Berendse HW, Scheltens P (2009) Graph theoretical analysis of magnetoencephalographic functional connectivity in Alzheimer's disease. Brain 132:213-224

Sutherling WW, Mamelak AN, Thyerlei D, Maleeva T, Minazad Y, Philpott L, Lopez N (2008) Influence of magnetic source imaging for planning intracranial EEG in epilepsy. Neurology 71:990-996

Taulu S, Simola J (2006) Spatiotemporal signal space separation method for rejecting nearby interference in MEG measurements. Phys Med Biol 51:1759-1768

Taulu S, Simola J, Kajola M, Helle L, Ahonen A, Sarvas J (2012) Suppression of uncorrelated sensor noise and artifacts in multi-channel MEG data. Poster in Biomag 2012, Paris

Thut G, Veniero D, Romei V, Miniussi C, Schyns P, Gross J (2011) Rhythmic TMS causes local entrainment of natural oscillatory signatures. Curr Biol 21:1176-1185

Vitikainen A-M, Lioumis P, Paetau R, Salli E, Komssi S, Metsähonkala L, Paetau A, Kičić D, Blomstedt G, Valanne L, Mäkelä JP, Gaily E (2009) Combined use of non-invasive techniques for improved functional localization for a selected group of epilepsy surgery candidates. NeuroImage 45:342-348

Zamrini E, Maestu F, Funke M, Mäkelä JP, Riley M, Bajo R, Sudre G, Fernandez A, Castellanos NP, Del Pozo F, Stam K, van Dijk B, Bagic A, Pekkonen E, Becker JT (2011) Magnetoencephalography (MEG) as a putative biomarker for Alzheimer's disease. Int $\mathbf{J}$ Alzh. Dis., doi:10.4061/2011/280289

Zhdanov A, Wilenius J, Paetau A, Ahonen A, Mäkelä JP (2013) Quantifying the contribution of video in combined video-magnetoencephalographic ictal recordings of epilepsy patients. Epilepsy Res 105:405-409 